\section{FREE SEMINARS TO KEEP YOU UP TO DATE}

Oral-B has updated the format of their popular 'Up To Date' seminars. Each evening session will now be comprised of three 35-minute lectures.

Professor Mike Lewis will be discussing the clinical presentation of mouth cancer and will be advising how to increase awareness of this devastating disease, in addition to improving the chances of its detection whilst the tumour is small. Professor Avi Banerjee will be presenting the third part of his 'MI' modern caries management trilogy, exploring the various restorative dental material options available to fill carious cavities with plenty of clinical illustrations and practical tips. Professor Iain Chapple will be looking into early diagnosis of periodontal and peri-implant diseases including screening in children and adolescents and will illustrate in a case-driven presentation the consequences of late diagnosis.

Dentists, hygienists and therapists are invited to attend this complimentary CPD-accredited evening event on the following dates: Birmingham (St John's Hotel Solihull, 12 Feb 2015); Newcastle (Hilton Gateshead, 26 Feb 2015); London (Shaw Theatre, Pullman Hotel Euston, 19 March 2015); Bristol (The Grand 'Thistle' Hotel, 23 April 2015); Glasgow (Westwood Hotel, 30 Apr 2015); Exeter (Exeter Racecourse, 21 May 2015); and Leeds (Village Hotel, 11 June 2015). Two and a half hours of verifia-

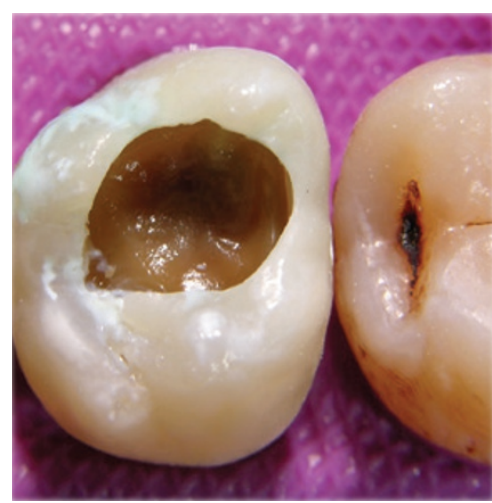

ble CPD and a complimentary meal are included. Spaces are allocated on a first come, first served basis. Register online at www.dentalcare. co.uk/uptodateseminars.

\title{
HELP PATIENTS MANAGE THEIR OWN MOUTHS
}

Dentisan is delighted to announce the launch of a new one-day course specifically designed to educate the whole dental team about every aspect of infection prevention and control. The courses, starting in November 2014, will be held once a month at Dentisan's brand new, purpose-built Centre for Decontamination Education (CDE) at their headquarters in Derby.

This course is unlike any other currently available in the sector and combines theoretical learning with hands-on practical application of decontamination procedures. The course will provide successful delegates with a City \& Guilds accredited qualification, making it a valuable addition to an individual's $\mathrm{CV}$, as well as giving important training to fulfil the role of Decontamination Lead, in accordance with the requirements of HTM 01-05. Furthermore, delegates registered with the GDC can claim up to 5.5 hours of verifiable core CPD.

Confirmed dates include 4 December 2014, 8 January, 5 February, 5 March and 2 April 2015.

Each course is limited to 12 participants. For more information, or to book, call Dentisan on 01332268526 or email ly@ dentisan.co.uk.

\section{A REVEALING WINDOW INTO DENTAL DISEASE}

The intraoral camera has provided dentists and their patients with a revealing window into the realm of dental disease, conferring greater transparency and more lucid communication. Durr has consolidated a range of technologies to ensure this invaluable tool has its most sophisticated application yet.

One of the most

versatile aspects of the VistaCam iX is the inter-

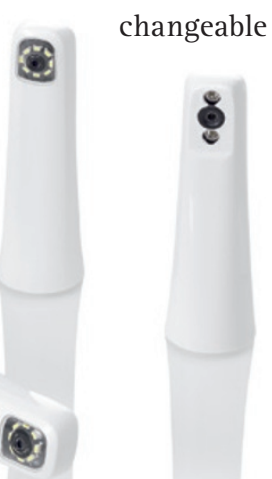

head mechanism. Four heads are available, one providing high-resolution images of the oral environment; a macro head for close-ups of up to $100 \times$ zoom; a Proof head for caries diagnosis with a colour-coded scale; and an LED curing light. Data transmission is fully digital through a USB port to a PC or can function as a standalone version without a PC. The unit also holds the promise of additional heads being added in the future, which will further enhance its range of applications.

The ergonomic head rotates a full 360 degrees to ensure that every part of the oral cavity is easily accessible, and a motion sensor automatically switches the camera on and off to ensure efficient usage. Its smooth finish enables easy disinfection and sheathing for cross infection. The VistaCam iX offers unrivalled functionality in a single device with multiple applications, perfectly complementing daily practice with an indispensable tool.
GC UK has put together Word of Mouth: a full colour brochure showing their latest promotions so that you can see the best prices for GC products and find out how GC products can benefit you and your patients.

GC prides themselves on their minimal intervention programme. It has been designed to reduce the loss of healthy tooth tissue, as well as helping patients to manage their own mouths with a variety of products such as Tooth Mousse sugar free topical paste, which provides extra protection for patients' teeth, and Tri Plaque ID Gel. This product allows you and your patient to identify areas of plaque in three easy steps.

Dentonauts are a family of characters that have been designed to help you encourage your younger patients to protect vulnerable erupting teeth with Fuji Triage. Fuji Triage is a selfadhesive material, is insensitive to moisture and has a low enough viscosity to penetrate pits and fissures, making it truly effective.

To receive your copy of Word of Mouth simply ask your local GC representative, or call GC directly on 01908218999. 